\title{
Geometry of an interplanetary CME on October 29, 2003 deduced from cosmic rays
}

\author{
T. Kuwabara, ${ }^{1}$ K. Munakata, ${ }^{1}$ S. Yasue, ${ }^{1}$ C. Kato, ${ }^{1}$ S. Akahane,,${ }^{1}$ M. Koyama, ${ }^{1}$ \\ J. W. Bieber, ${ }^{2}$ P. Evenson, ${ }^{2}$ R. Pyle, ${ }^{2}$ Z. Fujii, ${ }^{3}$ M. Tokumaru, ${ }^{3}$ M. Kojima, ${ }^{3}$ \\ K. Marubashi, ${ }^{4}$ M. L. Duldig, ${ }^{5}$ J. E. Humble, ${ }^{6}$ M. R. Silva, ${ }^{7}$ N. B. Trivedi, ${ }^{7}$ \\ W. D. Gonzalez, ${ }^{3,8}$ and N. J. Schuch ${ }^{7}$
}

Received 21 June 2004; revised 21 June 2004; accepted 24 August 2004; published 6 October 2004.

[1] A coronal mass ejection (CME) associated with an X17 solar flare reached Earth on October 29, 2003, causing an $\sim 11 \%$ decrease in the intensity of high-energy Galactic cosmic rays recorded by muon detectors. The CME also produced a strong enhancement of the cosmic ray directional anisotropy. Based upon a simple inclined cylinder model, we use the anisotropy data to derive for the first time the three-dimensional geometry of the cosmic ray depleted region formed behind the shock in this event. We also compare the geometry derived from cosmic rays with that derived from in situ interplanetary magnetic field (IMF) observations using a Magnetic Flux Rope model. INDEX TERMS: 2104 Interplanetary Physics: Cosmic rays; 2111 Interplanetary Physics: Ejecta, driver gases, and magnetic clouds; 2134 Interplanetary Physics: Interplanetary magnetic fields. Citation: Kuwabara, T., et al. (2004), Geometry of an interplanetary CME on October 29, 2003 deduced from cosmic rays, Geophys. Res. Lett., 31, L19803, doi:10.1029/ 2004 GL020803.

\section{Introduction}

[2] When a coronal mass ejection (CME) accompanied by a strong shock travels through interplanetary space, it often forms a depleted region of Galactic cosmic rays behind the shock and within the CME. When Earth enters the depleted region, ground-based cosmic ray detectors record a Forbush Decrease [Cane, 2000], often accompanied by strong enhancements of the cosmic ray anisotropy. By analyzing data recorded by a network of high latitude neutron monitors, Bieber and Evenson [1998] demonstrated that the temporal evolution of the cosmic rays is closely linked to magnetic properties of the $\mathrm{CME}$ and provides information on the structure of the CME as it approaches

\footnotetext{
${ }^{1}$ Physics Department, Shinshu University, Matsumoto, Japan. USA.

${ }^{2}$ Bartol Research Institute, University of Delaware, Newark, Deleware,

${ }^{3}$ Solar-Terrestrial Environment Laboratory, Nagoya University, Nagoya, Japan.

${ }^{4}$ National Institute of Information and Communications Technology, Tokyo, Japan.

${ }^{5}$ Australian Antarctic Division, Kingston, Tasmania, Australia.

${ }^{6}$ School of Mathematics and Physics, University of Tasmania, Hobart, Tasmania, Australia.

${ }^{7}$ National Institute for Space Research (INPE/OES/CRSPE), Sao Jose dos Campos, Santa Maria, Brazil.

${ }^{8}$ Now at National Institute for Space Research (INPE/OES/CRSPE), Sao Jose dos Campos, Santa Maria, Brazil.
}

Copyright 2004 by the American Geophysical Union. 0094-8276/04/2004GL020803\$05.00 and passes Earth. Munakata et al. [2004] confirmed this with observations by a network of muon detectors, which respond to higher energy primary cosmic rays $(\sim 60 \mathrm{GeV})$ than the neutron monitors $(\sim 10 \mathrm{GeV})$. In the present paper, we analyze muon data using an inclined cylinder to model the geometry of the cosmic ray depleted region. The cylinder is meant to represent a local section of a largescale loop structure draped from the sun by the CME [Ihara et al., 2003]. Preliminary results from earlier events can be found elsewhere [Munakata et al., 2003]; here, we apply an improved analysis method to the extreme event of October 29, 2003.

\section{Observations}

[3] Data recorded by three multi-directional muon detectors at Nagoya (Japan), Hobart (Australia), and São Martinho (Brazil) are analyzed here. For detailed properties of the detectors, we refer the reader to Munakata et al. $[2000,2001]$. We fit the function $I_{i, j}^{f i t}(t)$ given by

$$
\begin{aligned}
I_{i, j}^{f i t}(t)= & I(t) c_{0 i, j}^{0}+\xi_{x}^{G E O}(t)\left(c_{1 i, j}^{1} \cos \omega t_{i}-s_{1 i, j}^{1} \sin \omega t_{i}\right) \\
& +\xi_{y}^{G E O}(t)\left(s_{1 i, j}^{1} \cos \omega t_{i}+c_{1 i, j}^{1} \sin \omega t_{i}\right) \\
& +\xi_{z}^{G E O}(t) c_{1 i, j}^{0}
\end{aligned}
$$

to the pressure-corrected hourly count rates, $I_{i, j}^{o b s}(t)$, of cosmic ray secondary muons observed at universal time $t$ in the $\mathrm{j}$-th directional channel in the $\mathrm{i}$-th muon detector. This yields for each hour the best fit density of primary cosmic rays ( $I$, the omni-directional component of intensity) as well as the three components of the streaming vector, or first order anisotropy, in the geographic (GEO) coordinate system $\left(\xi_{x}^{G E O}, \xi_{y}^{G E O}, \xi_{z}^{G E O}\right)$. In equation $(1), t_{i}$ is the local time in hours at the $\mathrm{i}$-th station, $\omega$ is $\frac{2 \pi}{24}$, and $c_{0 i, j}^{0}, c_{1 i, j}^{1}$, $s_{1 i, j}^{1}$ and $c_{1 i, j}^{0}$ are so-called "coupling coefficients" which relate the observed muon intensity to the primary cosmic ray intensity in free space [Fujimoto et al., 1984]. We then transform the streaming vector to the geocentric solar ecliptic (GSE) coordinate system and subtract streaming due to solar wind convection and due to Earth's $30 \mathrm{~km} / \mathrm{s}$ motion about the Sun, yielding the anisotropy in the solar wind frame $\xi^{w}$. In these Compton-Getting subtractions, we assume the cosmic ray energy $(E)$ spectrum varies as $E^{-2.7}$, and for solar wind speed we employ the hourly mean bulk speed of alpha particles, as the proton bulk speed is unavailable for much of this event (http://umtof.umd.edu/ pm/speeds_302-304.gif). In the following analysis, we lag the ACE wind speed and magnetic field data by 20 minutes 


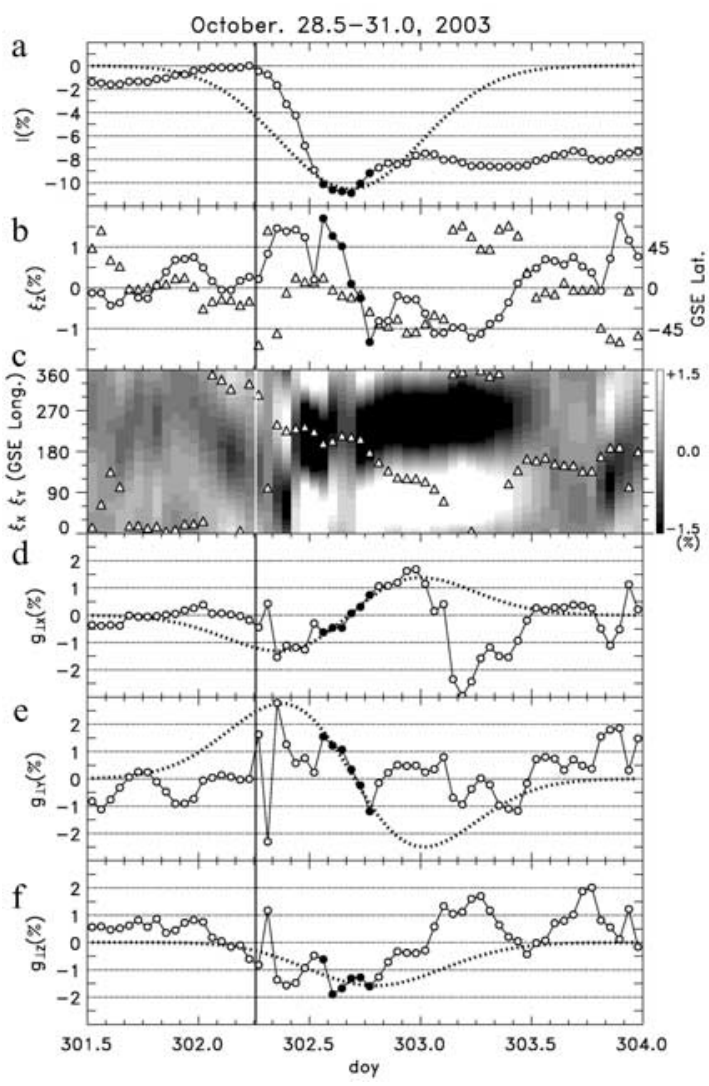

Figure 1. Cosmic ray event on October 29, 2003 observed by the muon detector network. Panels display as a function of time (day of year 2003) (a) the best fit density $I$ (circles), (b) north-south anisotropy $\xi_{z}$ (circles), (c) the component anisotropy in the ecliptic plane $\xi_{x}, \xi_{y}$ in a gray-scale format, and three components of the perpendicular density gradient (d) $g_{\perp x}$, (e) $g_{\perp y}$, and (f) $g_{\perp z}$ in GSE coordinates (circles). In Figure 1c, each vertical stripe represents 1 hour of data, with lighter and darker colors respectively denoting higher and lower cosmic ray intensity. Saturation occurs at $+1.5 \%$ (white) and $-1.5 \%$ (black). In Figures $1 \mathrm{~b}$ and $1 \mathrm{c}$, triangles show respectively the GSE latitude and longitude of the hourly mean IMF. The time of a Storm Sudden Commencement (SSC) is indicated by the vertical line. Closed circles in any panel denote the time interval used for the cylinder analysis, and the density and gradient vector reproduced by the best fit cylinder model are shown by dotted curves.

as a rough correction for the solar wind transit time between $\mathrm{ACE}$ and Earth.

[4] Following Bieber and Evenson [1998], we assume that enhanced anisotropy perpendicular to the IMF (B) is predominantly due to $\mathbf{B} \times \nabla N$ drift flux driven by a gradient of cosmic ray density $(N)$. Based upon this assumption, the fractional perpendicular density gradient is given by

$$
g_{\perp}(t)=R_{L} \frac{\nabla_{\perp} N}{N}=-\mathbf{b}(t) \times \boldsymbol{\xi}^{w}(t),
$$

where $R_{L}$ is the effective particle Larmor radius, and $\mathbf{b}(t)$ is a unit vector in the direction of $\mathbf{B}$.

[5] Figure 1 shows the cosmic ray event on October 29, 2003 observed by the muon detector network. Figure 1a shows that the cosmic ray density $I$ decreased by about $11 \%$ following shock arrival at the time of the SSC (vertical line). This is one of the largest decreases ever recorded by muon detectors. There is also a strong enhancement of the anisotropy as shown in Figures $1 \mathrm{~b}$ and 1c. Figure 1d shows that the GSE x-component $\left(g_{\perp x}\right)$ of the perpendicular gradient turns systematically from negative to positive, consistent with a cosmic ray depleted region approaching and then receding from Earth. On the other hand, the GSE z-component $\left(g_{\perp z}\right)$ remains negative, indicating the center of the depleted region passed north of Earth. The GSE y-component $\left(g_{\perp y}\right)$ turns systematically from positive to negative. These features of a CME passing Earth were first demonstrated from a high latitude neutron monitor network [Bieber and Evenson, 1998]. In the remainder of this paper, we develop a technique for analyzing cosmic ray data using a cylinder model for the CME, and we apply it to the extreme event on October 29, 2003.

\section{Analysis and Results}

\subsection{Cylinder Model for Cosmic Rays}

[6] We assume an axisymmetric spatial distribution for the cosmic ray density with a minimum located along the axis of a straight "cylinder," which is an idealized representation of a local section of a CME loop. In this model, the negative density gradient $\left(-\boldsymbol{g}_{\perp}(t)\right)$ observed at Earth is perpendicular to the cylinder axis and points toward the Closest Axial Point (CAP) on the cylinder axis. We assume a simple Gaussian function for the model density distribution,

$$
N(r)=N_{0}+n_{0} \exp \left(-\frac{r^{2}}{2 \lambda^{2}}\right)
$$

where $N_{0}$ is the background density, $n_{0}(<0)$ is the density depression on the cylinder axis, $r$ is distance between the CAP and Earth, and $\lambda$ is a parameter representing the cylinder thickness. The fractional density depression $I(r)$ at $r$ is given by

$$
I(r)=\frac{N(r)-N_{0}}{N_{0}}=I_{0} \exp \left(-\frac{r^{2}}{2 \lambda^{2}}\right)
$$

with $I_{0}=\frac{n_{0}}{N_{0}}(<0)$. The fractional density gradient vector $\left(g_{\perp}(t)\right)$, which can be obtained from the anisotropy measurement at a position (r), is given by

$$
\begin{aligned}
\boldsymbol{g}_{\perp}(\mathbf{r}) & =R_{L} \frac{1}{N} \frac{d N}{d \mathbf{r}}=R_{L} \frac{\mathbf{r}}{\lambda^{2}} \frac{I_{0} \exp \left(-\frac{r^{2}}{2 \lambda^{2}}\right)}{1+I_{0} \exp \left(-\frac{r^{2}}{2 \lambda^{2}}\right)} \\
& \approx R_{L} \frac{\mathbf{r}}{\lambda^{2}} I_{0} \exp \left(-\frac{r^{2}}{2 \lambda^{2}}\right),
\end{aligned}
$$

where we used $\left|I_{0}\right|=\left|\frac{n_{0}}{N_{0}}\right| \ll 1$ to simplify the expression.

[7] As illustrated in Figure 2, we can determine the position vector of the CAP as viewed from Earth, $\mathbf{P}_{\mathbf{E}}(t)$, by solving equation (5) for $\mathbf{r}$ using the derived $\boldsymbol{g}_{\perp}(t)$ for each hour. The position vector is then given by

$$
\mathbf{P}_{\mathbf{E}}(t)=-\mathbf{r}(t)=\mathbf{V}_{\mathbf{a p p}}\left(t-t_{0}\right)+\mathbf{P}_{\mathbf{0}},
$$

where $\mathbf{V}_{\mathbf{a p p}}$ is the apparent velocity of the CAP, $\mathbf{P}_{\mathbf{0}}$ is its impact parameter, and $t_{0}$ is time of closest approach. 


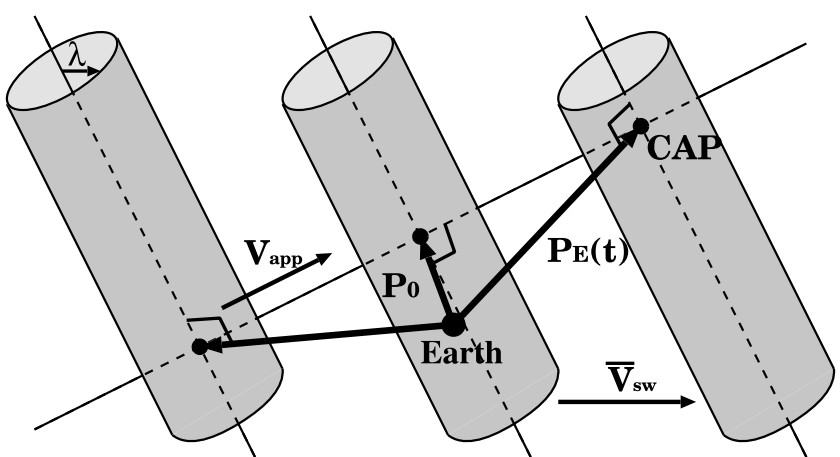

Figure 2. Geometry of an inclined cylinder passing Earth. The cylinder convects with the solar wind velocity $\overline{\mathbf{V}}_{\mathbf{s w}}$, but the closest axial point (CAP) to Earth has a different velocity $\mathbf{V}_{\text {app }}$. The position vector of the CAP is $\mathbf{P}_{\mathbf{E}}(\mathbf{t})$, and $\mathbf{P}_{\mathbf{0}}$ is the impact parameter. Although the cylinder is shown with a sharp edge at radius $\lambda$, the model actually assumes a Gaussian shaped density suppression centered on the cylinder axis; see equation (4).

[8] In this paper, we assume the cylinder moves with constant velocity equal to the solar wind velocity $\overline{\mathbf{V}}_{\mathbf{s w}}$ averaged over the analyzed time period. The CAP velocity observed in the solar wind frame, $\mathbf{V}_{\mathbf{a p p}}^{\mathbf{a x i s}}$, is then

$$
\mathbf{V}_{\text {app }}^{\text {axis }}=\mathbf{V}_{\text {app }}-\overline{\mathbf{V}}_{\text {sw }} \text {. }
$$

This velocity is aligned with the cylinder axis; hence it defines the orientation of the cylinder.

\subsection{Best Fitting to the Data}

[9] In the actual best fitting analysis, we use equations (4), (5), and (6) rewritten in terms of scale quantities normalized by $\lambda$, as

$$
\begin{gathered}
I(t)=I_{0} \exp \left(-\frac{1}{2} \rho_{E}(t)^{2}\right), \\
g_{\perp}(t)=-r_{L} \boldsymbol{\rho}_{E}(t) I_{0} \exp \left(-\frac{1}{2} \rho_{E}(t)^{2}\right), \\
\boldsymbol{\rho}_{E}(t)=\mathbf{v}_{\text {app }}\left(t-t_{0}\right)+\boldsymbol{\rho}_{0},
\end{gathered}
$$

where

$$
r_{L}=\frac{R_{L}}{\lambda}, \boldsymbol{\rho}_{E}(t)=\frac{\mathbf{P}_{\mathbf{E}}(t)}{\lambda}, \quad \mathbf{v}_{\mathbf{a p p}}=\frac{\mathbf{V}_{\text {app }}}{\lambda}, \boldsymbol{\rho}_{0}=\frac{\mathbf{P}_{\mathbf{0}}}{\lambda} .
$$

[10] We first choose a pair of parameters $I_{0}$ and $r_{L}$ and solve equation (9) for $\rho_{\mathbf{E}}(t)$ every hour. We then calculate $\mathbf{v}_{\text {app }}, \rho_{\mathbf{0}}$ and $t_{0}$ by fitting a straight line to each GSE component of $\rho_{\mathbf{E}}(t)$ plotted as a function of time $t$. With these parameters known, we compute the expected density $I^{\exp }(t)$ and gradient vector $\boldsymbol{g}_{\perp}^{\exp }(t)$, and we determine the residual $S$ defined by

$$
S=\sqrt{\frac{1}{4 N} \sum_{i=1}^{N}\left\{\left|I^{o b s}\left(t_{i}\right)-I^{\exp }\left(t_{i}\right)\right|^{2}+\left|\boldsymbol{g}_{\perp}^{\text {obs }}\left(t_{i}\right)-g_{\perp}^{\exp }\left(t_{i}\right)\right|^{2}\right\}}
$$

where $N$ is the total number of hours analyzed. We repeat these calculations changing $I_{0}$ and $r_{L}$, and we determine the best fit pair that minimizes $S$.

[11] The magnitude of $\mathbf{V}_{\text {app }}$ can now be calculated from

$$
\left|\mathbf{V}_{\text {app }}\right|=\left|\overline{\mathbf{V}}_{\mathbf{s w}}\right| \cos \Theta,
$$

where $\Theta$ is the angle between $\overline{\mathbf{V}}_{\mathbf{s w}}$ and $\mathbf{v}_{\text {app }}$ for the best fit pair. Using $r_{L}$ and $\rho_{0}$ corresponding to the best fit pair, $\lambda$ and the other parameters are then determined as follows,

$$
\lambda=\frac{\left|\mathbf{V}_{\text {app }}\right|}{\left|\mathbf{v}_{\text {app }}\right|}, R_{L}=r_{L} \lambda, \quad \mathbf{V}_{\text {app }}=\mathbf{v}_{\text {app }} \lambda, \mathbf{P}_{\mathbf{0}}=\rho_{0} \lambda .
$$

\subsection{Result}

[12] The best fit $I^{\exp }(t)$ and $\boldsymbol{g}_{\perp}^{\exp }(t)$ are shown by the dotted curves in Figures $1 \mathrm{a}$ and $1 \mathrm{~d}-1 \mathrm{f}$. The best fit calculations are performed over a time interval $\Delta t=6$ hours from 13:00-19:00 UT on 29 October 2003, shown by solid circles. The temporal evolution both of the density and the gradient are well reproduced even with such a simple model. The best fit parameters are given in Table 1. This analysis suggests that the cosmic ray cylinder in this event was inclined at latitude $\theta=3^{\circ}$ from the ecliptic and at GSE longitude $\phi=27^{\circ}$, as illustrated in Figure 3 (left). The scale size $\lambda$ of the cylinder is $\sim 0.1 \mathrm{AU}$, which corresponds to a FWHM of 0.28 AU. Closest approach was at 16:27 UT, at which time the CAP passed $\sim 0.035$ AU north of Earth.

\section{Discussion and Conclusion}

[13] We have derived for the first time the 3D geometry of a cosmic ray depleted region formed behind a strong interplanetary shock, which arrived at Earth on 29 October 2003 , by using the cosmic ray intensity observed with a network of muon detectors. This event caused a $\sim 11 \%$

\begin{tabular}{|c|c|c|c|c|c|c|c|c|c|c|c|}
\hline$\Delta t[$ hour $]$ & $\bar{V}_{s w}[\mathrm{~km} / \mathrm{s}]$ & $\bar{B}[\mathrm{nT}]$ & $I_{0}[\%]$ & $\lambda[\mathrm{AU}]$ & $\mathbf{V}_{\text {app }}[\mathrm{km} / \mathrm{s}]$ & $\mathbf{P}_{\mathbf{0}}[\mathrm{AU}]$ & $t_{0}[\mathrm{hh}: \mathrm{mm}]$ & $R_{L}[\mathrm{AU}]$ & $S[\%]$ & $\theta\left[{ }^{\circ}\right]$ & $\phi\left[^{\circ}\right]$ \\
\hline 6 & 1401 & 44 & -11.02 & 0.119 & $\begin{array}{c}-288.3 \\
561.7 \\
73.4\end{array}$ & $\begin{array}{c}-0.001 \\
-0.005 \\
0.036\end{array}$ & $16: 27$ & 0.056 & 0.342 & 3 & 27 \\
\hline
\end{tabular}
decrease in the omni-directional intensity of cosmic rays. By modeling the cosmic ray depleted region as a cylinder, we demonstrated that the observed systematic variation in the cosmic ray anisotropy is consistent with an inclined cylinder of thickness (FWHM) 0.28 AU approaching and then receding from Earth at the solar wind velocity of

Table 1. Best Fit Parameters for the Cylinder Analysis of October 29, 2003

\footnotetext{
${ }^{\mathrm{a}}$ See text for definitions. For vectors $\mathbf{V}_{\text {app }}$ and $\mathbf{P}_{\mathbf{0}}$, the 3 GSE components (x, y, z) are given.
} 
$\sim 1400 \mathrm{~km} / \mathrm{s}$. The center of the cylinder approached closest to Earth at 16:27 UT on 29 October, at which time it was $\sim 0.035$ AU north of Earth.

[14] The best fit Larmor radius $R_{L}$ in Table 1 is $0.056 \mathrm{AU}$. Given the observed IMF magnitude of $44 \mathrm{nT}$ averaged over the analysis period $(\bar{B})$, this corresponds to an effective rigidity of primary cosmic rays of $112 \mathrm{GV}$. This value is within the $60-120 \mathrm{GV}$ range of median primary rigidities covered by 39 directional channels in our present network [Munakata et al., 2004]. Noting that no information about the detector energy response was used in the cylinder analysis, the consistency of the inferred effective rigidity with the detector median rigidities supports the validity of the method.

[15] We also performed a best fit analysis of the Magnetic-Flux-Rope (MFR) model [Marubashi, 2000] to IMF data observed by ACE. Shown in Figure 4 are 64 second data of the IMF (a) magnitude, (b) latitude and (c) longitude together with the (d) solar wind velocity (helium bulk speed). The MFR model describes the data reasonably well, as indicated by good agreement between the data and solid curves. The 3-D geometry of the best fit MFR is depicted in Figure 3 (right). The inclinations of the MFR are $46^{\circ}$ latitude and $54^{\circ}$ longitude, which are in the same sense but somewhat larger than those of the cosmic ray cylinder $\left(3^{\circ}\right.$ and $\left.27^{\circ}\right)$. One reason for a difference is that the MFR analysis interval (delimited by solid vertical lines in Figure 4) is 2.5 times longer than that of the cosmic ray cylinder (dotted vertical lines). Extending the period of the cylinder analysis to later hours is problematic with the present simple model, however, because the fast decrease and slower recovery of the cosmic ray density cannot be modeled by a symmetric Gaussian. Keeping this restriction in mind, we extended the cylinder interval by two hours both earlier and later and obtained inclinations of $13^{\circ}$ latitude and $56^{\circ}$ longitude, closer to those of the MFR analysis, though the residual $S$ increased slightly to 0.457 from 0.342 in Table 1 . A second reason for a difference is that ACE samples the IMF only along a line determined by the solar wind flow, while the cosmic rays sample the magnetic field within a large region $\sim 0.1 \mathrm{AU}$ in diameter (two Larmor radii).

[16] For the future, it is important to develop more realistic (asymmetric) models of the cosmic ray depleted
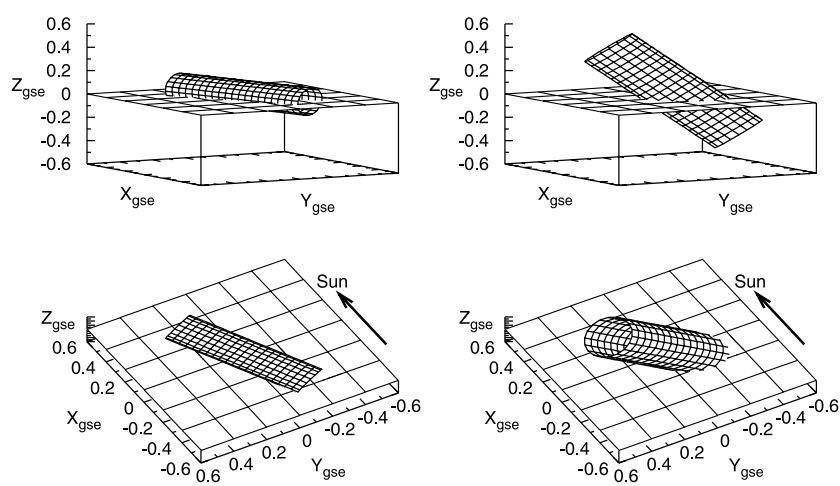

Figure 3. 3-D geometry in GSE coordinate system, as deduced from the cosmic ray cylinder model (left) and the Magnetic-Flux-Rope model (right).

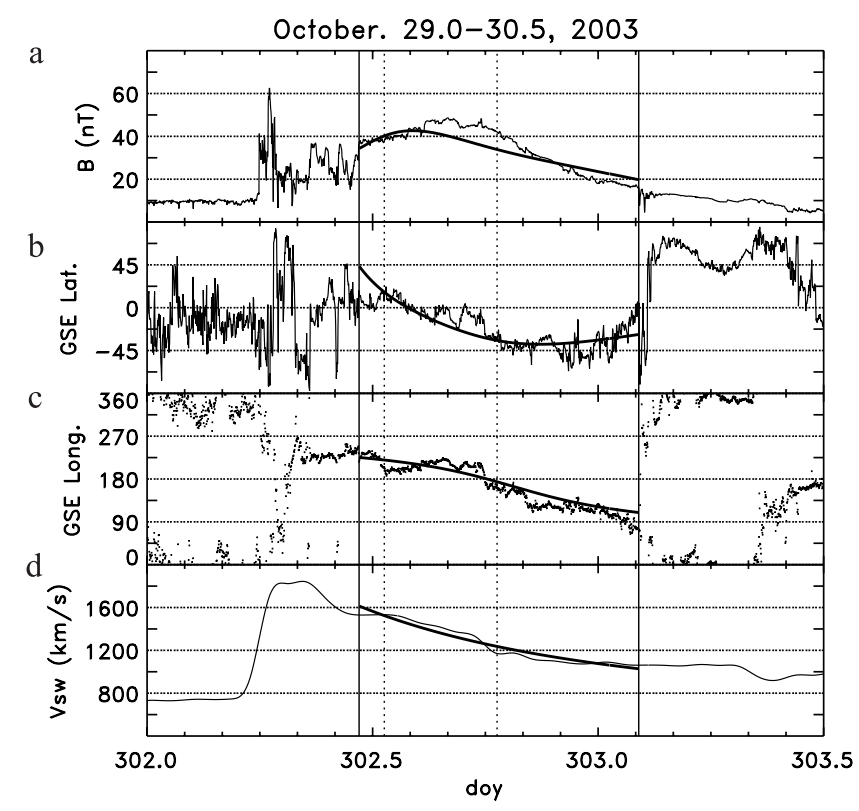

Figure 4. Temporal evolution of IMF parameters observed by ACE: 64-s data of IMF (a) magnitude, (b) latitude, (c) longitude, and (d) the solar wind speed. Period analyzed with the Magnetic-Flux-Rope model is delimited by solid vertical lines, and period analyzed with the cosmic ray cylinder model is delimited by dotted vertical lines. In this figure, IMF parameters are plotted against the observation time at ACE, while the cosmic ray analysis period indicated by the dotted lines is shifted 20 minutes earlier to correct for the solar wind transit time between ACE and Earth.

region. We will also work to enlarge the muon observing network in order to obtain a more complete picture of the cosmic ray angular distribution.

[17] Acknowledgments. This work is supported in part by U.S. NSF grants ATM-0207196 and ATM-0000315, and in part by a Grants-in-Aid for Scientific Research from the Ministry of Education, Culture, Sports, Science and Technology in Japan and by the joint research program of the Solar-Terrestrial Environment Laboratory, Nagoya University. We thank N. F. Ness for providing ACE magnetic field data via the ACE Science Center.

\section{References}

Bieber, J. W., and P. Evenson (1998), CME geometry in relation to cosmic ray anisotropy, Geophys. Res. Lett., 25, 2955-2958.

Cane, H. V. (2000), Coronal mass ejections and forbush decreases, Space Sci. Rev., 93, 55-77.

Fujimoto, K., et al. (1984), Coupling coefficients of cosmic ray daily variations for meson telescopes, Rep. 9, Cosmic Ray Res. Lab., Nagoya, Japan.

Ihara, A., et al. (2003), A global structure of the magnetic flux rope observed in interplanetary space fitted by a torus-type force-free model, in 28th International Cosmic Ray Conference, vol. 6, edited by T. Kajita et al., pp. 3557-3560, Univ. Acad. Press, Tokyo.

Marubashi, K. (2000), Physics of interplanetary magnetic flux ropes: Toward prediction of geomagnetic storms, Adv. Space Res., 26, 55-66.

Munakata, K., et al. (2000), Precursors of geomagnetic storms observed by the muon detector network, J. Geophys. Res., 105, 27,457-27,468.

Munakata, K., et al. (2001), A prototype muon detector network covering a full range of cosmic ray pitch angles, in 27th International Cosmic Ray Conference, vol. 9, edited by R. Schlickeiser, pp. 3494-3497, Copernicus, Katlenburg-Lindau, Germany.

Munakata, K., et al. (2003), CME geometry deduced from cosmic ray anisotropy, in 28th International Cosmic Ray Conference, vol. 6, edited by T. Kajita et al., pp. 3561-3564, Univ. Acad. Press, Tokyo. 
Munakata, K., et al. (2004), CME-geometry and cosmic-ray anisotropy observed by a prototype muon detector network, Adv. Space Res., in press.

S. Akahane, C. Kato, M. Koyama, T. Kuwabara, K. Munakata, and S. Yasue, Physics Department, Shinshu University, Matsumoto 390-8621, Japan. (kuma00@gipac.shinshu-u.ac.jp)

J. W. Bieber, P. Evenson, and R. Pyle, Bartol Research Institute, University of Delaware, Newark, DE 19716, USA. (john@bxclu.bartol. udel.edu)
M. L. Duldig, Australian Antarctic Division, Kingston, Tasmania 7050, Australia.

Z. Fujii, M. Kojima, and M. Tokumaru, Solar-Terrestrial Environment Laboratory, Nagoya University, Nagoya, Aichi 464-8601, Japan.

W. D. Gonzalez, N. J. Schuch, M. R. Silva, and N. B. Trivedi, Southern Regional Space Research Center, National Institute for Space Research (OES/CRSPE/INPE), 97110-970 Santa Maria RS, Brazil.

J. E. Humble, School of Mathematics and Physics, University of Tasmania, Hobart, Tasmania 7001, Australia.

K. Marubashi, National Institute of Information and Communications Technology, Koganei, Tokyo 184-8795, Japan. 